anaesthetists ${ }^{6}$ and heard how one major department is experimenting with the use of personality questionnaires and a trained psychologist in the selection process for new entrants to the specialty.

Some easily avoidable tragedies occur at the hands of trainees, and over the years there has developed a nagging unease that the examination hurdles of the Faculty of Anaesthetists are inappropriate to the natural order of training in the specialty. The changes which will come into effect in 1985 are designed to focus the attention of the first year trainee on safety and craft competence before encouraging him to learn the more detailed aspects of pathophysiology, pharmacology, and the physical sciences necessary for the fully trained specialist. ${ }^{7}$ The consequent need to reappraise the influence of National Health Service gradings on the timing of examination hurdles was also touched on.

A topic that was highlighted for further attention was the effect of the quality of management and administration in anaesthetic departments and the importance of efficient scheduling of both work and training. For example, there is a need to mitigate the tensions created when a slow surgeon or an over running operating list threatens to impinge on some other scheduled commitment, whether professional or social. Interestingly, anaesthetics is the first specialty in which a course on management has been specifically designed solely for its members. Another aspect of the possible influence of departmental organisation on the quality of care is the need to rethink what the emergency anaesthetist could be doing while waiting for the emergency. Given reliable communications, could he not be attending to the quality of postoperative pain relief, generally acknowledged to be a major weakness in modern medical practice ? ${ }^{8}$

In fact, relatively few totally new ideas emerged that might have a direct impact on safety or on the quality of care. Perhaps this is because in terms of the usual indicators we are now working on a vanishingly small margin. The number of avoidable deaths is a very small proportion of the total number of anaesthetics given and may not be reducible by general educational efforts. It is probably still as true today as when first written that: "In the great majority [of deaths] there was a serious departure from generally accepted safe practice." Critical incident reporting, recently introduced by the British Airline Pilots Association, may have a more relevant part to play.$^{10}$ At the other end of the range much minor morbidity is knowingly accepted for the sake of other benefits. ${ }^{11}$

From the patient's point of view, empathy and reassurance before the operation, good (and safe) pain control after it, and freedom from the ill effects of anaesthesia are his only possible measures of quality. These are a good deal less easy to measure than the indices on which we currently focus. The vigour with which the specialty, both corporately and individually, is addressing itself to these problems is perhaps merely the obverse of the difficulty of making rapid progress. At all events, and despite its problems, the book which was written (and rewritten) at this meeting promises to be a milestone in the pursuit of high quality care in anaesthesia.

M D VICKERS

Professor of Anaesthetics,

Welsh National School of Medicine,

Cardiff CF4 4XN

${ }^{1}$ Anonymous. Less listening, more discussion. Br Med $\mathcal{F} 1981 ; 283: 2$.

2 Dobbing J, ed. Maternal nutrition in pregnancy: eating for two? New York: Academic Press, 1981.

${ }^{3}$ Faculty of Anaesthetists. Quality control in anaesthesia. London: Royal College of Surgeons of England, 1983.

4 Anonymous. Help for the sick doctor. Anaesthesia 1979;34:410-1.
${ }^{5}$ Lunn JN, Mushin WW. Mortality associated with anaesthesia. London: Nuffield Provincial Hospitals Trust, 1982.

${ }^{6}$ Reeve PE. Personality characteristics of a sample of anaesthetists. Anaesthesia $1980 ; 35: 559-68$.

${ }^{7}$ Faculty of Anaesthetists. Guidance on examinations leading to $D A(U K)$ and FFARCS (Eng). London: Royal College of Surgeons of England, 1983.

${ }^{8}$ Anonymous. Postoperative pain. $\mathrm{Br} \mathrm{Med} \mathcal{O}$ 1978;ii :517-8.

${ }^{9}$ Edwards G, Morton HJV, Pask EA, Wylie WD. Deaths associated with anaesthesia; a report on 1000 cases. Anaesthesia 1956;11:194-220.

${ }^{10}$ Craig J, Wilson ME. A survey of anaesthetic misadventures. Anaesthesia $1981 ; 36$ :933-6.

$"$ Goold JE. Anaesthesia for day-care surgery; a review. $\mathcal{f} R$ Soc Med 1983; 76:415-20.

\section{Changing patterns of cervical cancer rates}

Despite all the efforts put into screening for cancer of the cervix mortality from the disease in England and Wales has changed very little in the past 15 years. This apparent lack of effect on mortality rates has raised questions about the effectiveness of the cervical screening programme: doubts have been expressed both about the official policy of concentrating screening on older women ${ }^{1}$ and about the actual practice in which more intensive screening appears to have been carried out on younger women ${ }^{2-4}$ and on those of higher social class. ${ }^{4}$

Evidence for the effectiveness of screening is available from a number of other countries. Hakama ${ }^{6}$ has summarised recent data from the Nordic countries, concluding that changes in incidence in these countries have corresponded with the level of screening, the effect being most pronounced in Iceland, which has the most intensive screening, followed by Finland and Sweden. In each of these countries screening covers the entire country, but in Finland and Sweden the age range is shorter than in Iceland, and screening is repeated at longer intervals. A smaller effect was observed in Denmark, where only $40 \%$ of women are covered by an organised screening programme; in Norway, where the organised programme covered only $5 \%$ of the population, the disease showed an increasing trend during the period considered. Hakama suggests that the use of personal invitations to take part in screening programmes is an important factor in bringing women at high risk into the screening programme and hence in the reduction in incidence.

Miller et $a l^{7}$ found evidence for a relation between the intensity of screening and the fall in death rates from cancer of the uterus in the 10 provinces of Canada between 1960-2 and 1970-2. In a later analysis, however, Miller et al ${ }^{7 \mathrm{a}}$ found no correlation between intensity of screening and changes in mortality over the period 1964-6 to 1974-6; one possible explanation is that the women most at risk were not being covered by the screening programme even though the number of smears was increasing. $\mathrm{Cramer}^{8}$ showed that falls in mortality in different areas of the United States were also related to the level of screening. Such relations are not, of course, necessarily causal. Macgregor and Teper $^{9}$ analysed mortality data for the years 1968-76 in Scotland, comparing the Grampian and Tayside regions-where screening was most intensive-with the rest of Scotland, and concluded that trends in mortality in these regions were attributable to the screening programme, though the results were based on small numbers.

Various explanations have been suggested for the apparent 
failure of screening in England and Wales. If the screening programme is indeed ineffective the most obvious explanation is that the test is unreliable (which seems improbable in view of the experience of other countries) or that acceptance rates may have been low among high risk groups. It is also possible, however, that screening has in fact been effective but that the results have been obscured by an increase in disease frequency, particularly among younger women; trends in registration and mortality rates suggest that this may indeed be the case. Additional evidence that such an increase may have occurred comes from reports that the detection rate of "positive" smears has been increasing ( $\mathrm{p}$ 526). ${ }^{1011}$

The age specific death rates from cervical cancer showed considerable variations during the period 1965-81. One of the most striking has been the increase in recent years in death rates among women aged below 35: the rates at ages 25-29 and 30-34 doubled in 10 years, though the actual number of women dying under the age of 35 is still only about 120 a year-less than $6 \%$ of all deaths from cervical cancer. At ages 35-39 a decline occurred up to about 1970 , but the rates in this age group have increased by around $60 \%$ in the past 10 years. There has also been an increase in the age group 40-44 but this has lagged about five years behind that for the age group 35-39. Death rates at ages 45-49 and 50-54 have decreased considerably, while those at higher ages have decreased or remained roughly the same.

Similar variations have occurred in registration rates for invasive cancer between 1965 and 1979-the latest year for which registration data are available. ${ }^{12}$ In 10 years these rates have doubled for the age group 20-24 (though there are still fewer than 50 cases a year in this age group) and for ages 25-29 and 30-34. At ages 35-39 the rates fell for some years after 1965 but have more recently been increasing. These changes in registration rates and alsc those at higher ages are similar to the pattern of mortality described above.

These changes in registration and mortality rates are the net results of changes in disease rates, the effect of screening, and of other factors such as improved survival, hysterectomy rates, and changes in diagnostic criteria. Changes in mortality rates have occurred at different times for different age groups. As was originally pointed out by Hill and Adelstein, ${ }^{13}$ there is an important "birth cohort" effect in the pattern of mortality rates for cervical cancer; the same is true of registration rates. In England and Wales, for instance, women born in years around 1921 have generally high rates, while those born around 1931 have low rates. The striking increase in rates for women born around 1951 and later as compared with those born in earlier years suggests that these women would be expected to have high rates throughout their life span.

Information on the extent and effects of the screening programme is incomplete and difficult to interpret. Large increases have also occurred in registration rates for carcinoma in situ in younger women: to some extent these are simply a reflection of an increase in screening and may also be affected by changes in the completeness of registration, but it seems clear that there has been a substantial increase in the true incidence of carcinoma in situ. During 1973-9 the estimated annual number of smears as notified to the Department of Health and Social Security increased by only about $18 \%$-from 2.34 million to $2 \cdot 75$ million a year. ${ }^{10}$ Furthermore, it is estimated that more than $60 \%$ of smears taken during 1979 were examinations for women who had been previously screened (unpublished data provided by the DHSS). Thus the registration rate for carcinoma in situ might have been expected to decrease, since many of the "positives" should have been removed through detection at an earlier screening. Yet between 1973 and 1979 the rate actually increased by about $60 \%$ for women aged 15-24 and doubled for those aged 25-34. ${ }^{12}$ Rates of carcinoma in situ per 1000 smears taken have also been increasing, the sharpest increases occurring among women aged 25-29 and 30-34. In these two age groups the total number of smears increased by only $11 \%$ between 1973 and 1979, whereas the number of cases of carcinoma in situ registered increased by $117 \%$.

Plainly the increase in registration rates cannot be accounted for by increases in the numbers of smears taken but suggests that there has been a true increase in premalignant lesions in recent years, at least among younger women. There are other possible explanations: a greater proportion of women at high risk may have come forward for screening in recent years, or the interval between smears may have lengthened so increasing the chance of new lesions arising, or pathologists may have tended increasingly to diagnose "carcinoma in situ" where previously they would have applied the label "dysplasia." It seems improbable, however, that these factors would be concentrated among younger women and that the rates would have continued to increase to the extent observed.

In our view the most reasonable interpretation of the mortality, registration, and screening data-one which is consistent with what is known about the epidemiology of cervical cancer-is that there has been among younger women a very substantial increase in the rates of carcinoma in situ which, together with the smaller increases in those for invasive cancer, suggest that screening is holding in check a much larger potential increase in the rates of invasive disease among these younger women. Additional, though rather limited, evidence for an effect of screening comes from a more detailed analysis of the cohort data referred to above, in that some of the mortality rates after the introduction of the screening programme are lower than would have been predicted from the rates for younger women in the same cohorts.

Ideally, in any discussion of screening policy we should try to estimate the number of lives saved and the decrease in morbidity attributable to various alternative policies and choose a policy on the basis of the costs and benefits, taking into account other ways in which the money might be spent and the benefits thereby achieved. In practice a cervical cancer screening programme cannot be evaluated in this way, since the information necessary for making the comparisons is not available. The best we can do is to assume that the number of smears to be taken in each year is fixed and then consider the most effective way of distributing this effort on the basis of reasonable assumptions about the natural history of cervical neoplasms and the effectiveness of screening. The recommended policy for England and Wales ${ }^{3}$ explicitly acknowledges the possibility that the incidence among younger women may be increasing. It does not necessarily follow, however, nor is it recommended as part of the policy, that screening should be concentrated among younger women, since only a small proportion of invasive cervical cancer occurs among such women. Furthermore, the observed increase may be the first sign of a cohort effect that will be observed throughout the life span of women born in the early 1950s and, perhaps, more recently. Though high rates of carcinoma in situ are being observed among younger women, it is assumed that large numbers of these cases could be detected several years later (before developing into invasive cancer); indeed some might never progress.

The proposed policy is that the screening effort among younger women should be continued but at a lower frequency 
-a policy of annual screening cannot be justified with currently available resources, if at all. Screening resources should be used to give a greater coverage of the total population among women of all ages. Population screening is proposed at five year intervals for women aged 35 or over. In addition any woman above this age who has never had a smear must be encouraged to have at least one. There is little doubt that this is the most important single measure which could be adopted in cervical cancer screening. Methods for calling and recalling women for screening should be established.

Predictions about possible changes in the recommended ages and frequency for screening are difficult to make. We have suggested above that there is no method of determining the optimum level of resources to be devoted to screening and that in practice the question becomes one of deciding how a given amount of screening effort should be distributed. If, as seems likely, however, the present increase among younger women continues through their life span, and if succeeding cohorts show similar increases, it may become necessary to recommend an actual increase in the level of screening.

Very careful monitoring of the screening programme and the rates of both preclinical and clinical disease will be required for the development of future policies on either the distribution of screening effort or the total level of resources to be devoted to it. It will therefore be necessary to ensure that well designed computerised information systems are set up which can provide the necessary data. The systems currently being developed should be coordinated and the records produced should be usable for population based analyses and for linkage with cancer registration data.

\section{G J DRAPER}

Director,

Childhood Cancer Research Group,

Radcliffe Infirmary,

Oxford OX2 $6 \mathrm{HE}$

G A Cook

Senior Registrar in Community Medicine,

West Berkshire Health Authority,

Reading RG1 5LF

1 Yule R. Mortality from carcinoma of the cervix. Lancet 1978; :1031-2.

${ }^{2}$ Adelstein AM, Husain OAN, Spriggs AI. Cancer of the cervix and screening. Br Med f 1981 ;282:564.

${ }^{3}$ Draper GJ. Screening for cervical cancer: revised policy. The recommendations of the DHSS Committee on Gynaecological Cytology Health Trends $1982 ; 14: 37-40$.

- Wakefield J. The family doctor and cervical cytology. Health Trends 1971; 3:25-9.

${ }^{5}$ Parkin DM, Collins W, Clayden AD. Cervical cytology screening in two Yorkshire areas: pattern of service. Public Health 1981;95:311-21.

${ }^{6}$ Hakama $M$. Trends in the incidence of cervical cancer in the Nordic countries. In: Magnus $\mathrm{K}$, ed. Trends in cancer incidence. Causes and practical implications. Washington: Hemisphere Publishing Corporation, $1982: 279-92$.

${ }^{7}$ Miller AB, Lindsay J, Hill GB. Mortality from cancer of the uterus in Canada and its relationship to screening for cancer of the cervix. Int $\mathcal{f}$ Cancer 1976;17:602-12.

is Miller AB, Visentin T, Howe GR. The effect of hysterectomies and screening on mortality from cancer of the uterus in Canada. Int $\mathcal{F}$ Cancer $1981 ; 27: 651-7$.

${ }^{8}$ Cramer DW. The role of cervical cytology in the declining morbidity and mortality of cervical cancer. Cancer 1974 ;34:2018-27.

${ }^{9}$ Macgregor JE, Teper S. Mortality from carcinoma of cervix uteri in Britain. Lancet 1978;ii :774-6.

10 Roberts A. Cervical cytology in England and Wales, 1965-80. Health Trends 1982;14:41-3.

11 Bamford PN, Barber M, Beilby JOW. Changing pattern of cervical intraepithelial neoplasia seen in a family planning clinic. Lancet 1982;i:747.

12 Office of Population Censuses and Surveys. Cancer statistics: registrations 1979. London: HMSO (in press). (Series MBI, No 11.)

${ }^{13}$ Hill GB, Adelstein AM. Cohort mortality from carcinoma of the cervix. Lancet 1967 ;ii:605-6.

\section{Sleeping and dreaming}

"In spite of many thousands of years of effort," wrote Sigmund Freud in Die Traumdeutung, "the scientific understanding of dreams has made very little advance." That statement remained substantially true until the existence of two qualitatively different states of mammalian sleep was established 30 years ago. ${ }^{1}$ One of these states is associated with a relative slowing of the electroencephalogram, a quiescence of the autonomic nervous system, and no rapid eye movements or thoughtlike mental activity; the other is characterised by a desynchronised electroencephalogram, autonomic activation, rapid eye movements (hence the term rapid eye movement (REM) sleep), and dreaming.

This distinction has stimulated the interest of many scientific and clinical research workers in the mechanisms and functions of states of sleep, including Francis Crick, who, with Graeme Mitchison, has recently produced a characteristically provocative outline of a theory of dream sleep. ${ }^{2}$ Essentially, Crick and Mitchison have built on the observation that during REM sleep the brain, isolated from its input and output channels, receives non-specific signals from the brain stem. These, they suggest, "tune" the cortical system by removing unwanted or "parasitic" modes of neuronal interaction arising from either cerebral growth or experiential modifications. This is effected by a process of "reverse learning," which weakens the strength of synaptic associations and helps to eliminate inappropriate brain traces. In teleological terms, therefore, REM sleep becomes a "cleaning up mechanism," and "in REM sleep we unlearn our unconscious dreams." 2 The prime function of dreams, most of which we do not remember, is to forget. The CrickMitchison theory therefore assigns them a much more humble role than that of Freud's royal road to the knowledge of the unconscious activities of the mind. The biological findings cannot be regarded as the correlates of Freudian theory.

Crick and Mitchison maintain that their theory is consonant with a large body of experimental data, such as the prominence of REM sleep in early life and the hallucinatory nature of dreams during REM sleep. They also admit, however, that it does not explain either the effects of deprivation of REM sleep or the prevention of such sleep by drugs, with psychological ill effects. As the possibility of directly testing a reverse learning mechanism appears to be remote in our present state of knowledge, the possibilities of indirect methods demand exploration. One such method is mathematical modelling, which has already been used by Hopfield et al to study "unlearning" in a collective neural network of 30-1000 neurones. ${ }^{3}$ Another is the study of comparative biology, with particular reference to Tachyglossus aculeatus (the spiny anteater)-a creature that possesses a relatively large neocortex but does not exhibit REM sleep. According to Crick and Mitchison, the reason for this discrepancy may be that these mammals need a cortex of this size precisely because they are unable to "tune it up" by reverse learning.

As the authors freely admit, their views must be regarded as speculative at this stage. But we know something of the track record of the senior author, whose scientific method of working has been described as that of a man "who occasionally did experiments but more often was immersed in ... theories. ... Often he came up with something novel, would become enormously excited, and immediately tell it to anyone who would listen. A day or so later he would often realise that his theory did not work and return to experiments, until boredom generated a new attack on theory." This memorable 